\title{
The Effect of Tomography Imaging Artefacts on Structural Analysis and Numerical Permeability Simulations
}

\author{
Viivi Koivu and Tuomas Turpeinen \\ University of Jyväskylä, Department of Physics \\ Finland
}

\section{Introduction}

Fluid flow phenomena in porous materials can be found in many important processes in nature and in society. In particular, fluid flow through a porous medium contribute to several technological problems, e.g. extraction of oil or gas from porous rocks, spreading of contaminants in fluid-saturated soils and certain separation processes, such as filtration (Torquato, 2001). In paper and wood industry single and multi phase fluid flow properties in porous media play important roles related to manufacturing process and product development.

The general laws describing creeping fluid flows are well known. However, a detailed study of fluid flow in porous heterogeneous media is complicated. This is a direct consequence of the often very complex, internal micro-scale structures of these materials. That is, the interplay between fluid flow and complex internal structure at the micro-scale gives rise to the effective fluid flow properties at the macro-scale. Traditionally, efforts for analysing fluid flow properties by means of modelling are based on using regular pore geometries that may possess the bulk properties of the actual medium and are simple enough to allow for analytic solution of the relevant transport equations. However, the development of imaging techniques based on computerised x-ray micro-tomography $(\mathrm{C} X \mu \mathrm{T})$ together with advanced numerical techniques have made it possible to analyse structural and transport properties of complex materials based on 3D digitalisation of their real microstructures (Coles et al., 1998; Samuelsen et al., 2001; Goel et al., 2002; Thibault \& Bloch, 2002; Holmstad et al., 2003; Rolland et al., 2005; Goel et al., 2006; Stock, 2009). X-ray tomography is a non-invasive and non-destructive imaging method where individual $x$-ray images recorded from different viewing directions are used for reconstructing the internal 3D structure of the object of interest (Stock, 2009). Although a great opportunity to materials research, $\mathrm{CX} \mu \mathrm{T}$ also poses new challenges. The imaging method produces noise, edge blurring, and various other artefacts that may distort the $3 \mathrm{D}$ reconstruction of the sample structure and thus result in unrealistic analysis results.

In various industrial and scientific applications an effective material property, permeability, is used for describing the ability of porous materials to transmit fluids. Permeability coefficient for single phase creeping fluid flow through a porous media is defined by the phenomenological law by Henry Darcy as the proportionality constant between the average fluid velocity and applied pressure gradient (Darcy, 1856). The analytical approaches to analyse permeability are often confined to simplified sample geometries. Some of the 
numerical permeability studies are based on analysis made for computationally generated models of porous media (Rasi et al., 1999; Aaltosalmi et. al., 2004; Belov et al., 2004; Holmstad et al. 2005; Lundstrom et al., 2004; Verleye et al., 2005; Verleye et al., 2007). Tomographic reconstructions are increasingly utilised in combination with numerical methods to analyse permeability of porous materials (Manwart et al., 2002; Martys \& Hagedorn, 2002; Aaltosalmi et al., 2004; Kutay et al., 2006; Fourie et al., 2007). According to our knowledge, only a few studies are based on analysing the effect of tomographic image properties on numerical permeability results (Aaltosalmi et al., 2004; Holmstad, 2005)

The effects of imaging noise, imaging artefacts and the quality of image segmentation on flow permeability found by using direct numerical flow simulation by a specific implementation of finite-difference method (FDM) (Wiegmann, 2007) are studied. The specific surface area of the samples and the features of pore geometry are also analysed. The analyses are done for four different sample types. First, an artificial sample geometry, comprised of hexagonal array of cylinders with known analytical permeability result, is used to analyse the effect of added random noise and edge blurring on the analysis results. Second, $\mathrm{CX} \mu \mathrm{T}$ reconstructions of wool fibre web, packaging board and sandstone samples are used to illustrate the effects of different artefact removal and image segmentation methods on permeability results. Finally, the numerically simulated values of flow permeability are compared with experimental results for the same material.

\section{Tomography and image processing}

$\mathrm{CX} \mu \mathrm{T}$ is a non-destructive technique for analysing interior features within solid objects and for obtaining digital information of their 3D structure and properties. During the recent decade, the precision of $x$-ray tomographic imaging techniques have reached the submicrometre resolution and enables analyses of statistical properties of various materials.

\subsection{Tomography imaging of porous samples}

Authentic simulation geometries were obtained by utilising $\mathrm{CX} \mu \mathrm{T}$. Both synchrotron -based $x$-ray beams and conventional $x$-ray tubes were used as the radiation sources. The adequate resolution and the overall quality of the images depend on the techniques used. In this study, two laboratory scale devices (Sky-Scan 1172 and Xradia Micro XCT-400) based on xray tubes, and a tomographic imaging facility ID19 of the European Synchrotron Radiation Facility (ESRF) were used.

The voxel resolution of the laboratory scale devices can be varied from a few micrometres up to few tens of micrometres. The wool fibre web sample was imaged by SkyScan 1172 device with the voxel resolution of $4.48 \mu \mathrm{m}$ and the sand stone sample with Xradia Micro XCT-400 with the voxel resolution of $2.10 \mu \mathrm{m}$. The packaging board sample was scanned at ESRF beam-line ID19. The imaging set-up used at ID19 facility had the voxel resolution fixed to $0.7 \mu \mathrm{m}$.

\subsection{Imaging artefacts and image processing}

$\mathrm{CX} \mu \mathrm{T}$ imaging is a two phased process. First, a tomographic scanner is utilised to acquire a series of 2D shadowgraphs of a sample from multiple angles. Then, the 3D reconstruction of the sample is computed from the shadowgraphs using special algorithms (Kak \& Slaney, 1988). The process involves many sources of artefacts.

Typical CX $\mathrm{CT}$ imaging system is comprised of a light source, optical elements and a camera which all pose certain imaging artefacts (Gonzales \& Woods, 2002). Basically, the main 
imaging artefacts are noise and edge blurring caused by optics or the non-optimal light source of laboratory scale devices. In addition, the reconstruction procedure can add more artefacts to the tomographic reconstructions (Stock, 2009). The main reconstruction -based artefacts are rings, streaks and shadows caused by hardening of x-rays (Stock, 2009) or mechanical inaccuracy of the system.

Many image processing tools have been developed in order to overcome the problems related to the imaging artefacts, see e.g. Stock (2009) and references therein. However, most of the artefacts cannot be fully removed algorithmically and they thus require either removal by hardware optimisation or robust analysis software.

\subsubsection{Sample geometry preparation}

Representative elementary volumes (REVs) of the sample geometries were cropped out of the full $\mathrm{CX} \mu \mathrm{T}$ reconstructions. In this study the REVs were determined in a deterministic way by evaluating the porosity of larger and larger sample volumes always centred on the same image voxel (Drugan \& Willis, 1996; Rolland du Roscoat et al., 2007). A REV size thus obtained for the wool fibre web sample was (in XxYxZ -directions, see Fig. 1) 300x300x360 voxels, for the sand stone sample 500x500x500 voxels and for the packaging board sample $450 \times 450 \times 420$ voxels.

The sample REVs were filtered by variance-weighted mean filter (Gonzales \& Woods, 2002) and later thresholded to yield binary images including the solid material and the pore space. Visualisations of the REVs are presented in Fig. 1.

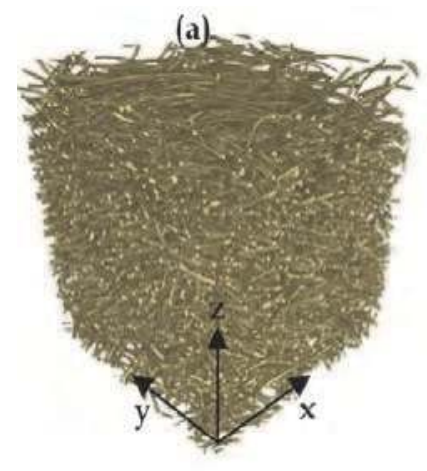

Fig. 1. Tomographic reconstructions of (a) the wool fibre web, (b) the packaging board and (c) the sandstone samples.

\subsubsection{Edge blurring and imaging noise}

Especially in laboratory scale CX $\mu \mathrm{T}$ systems, like SkyScan and Xradia, the image quality of the material edges in the reconstructed geometry is limited by optical properties of the system. The edge spreading is caused by the non-zero aperture diameter of the $x$-ray source, the optics in between the source and detector, and scintillator, i.e. the component that converts the $x$-rays into visible light. Thus, instead of sharp transition between different material phases, there is a smooth curve called edge spread function (ESF). In Fig. 2, the edge smoothness can be seen in the intensity profile plot. The width of the ESF in this case is around 7 pixels. 
(a)

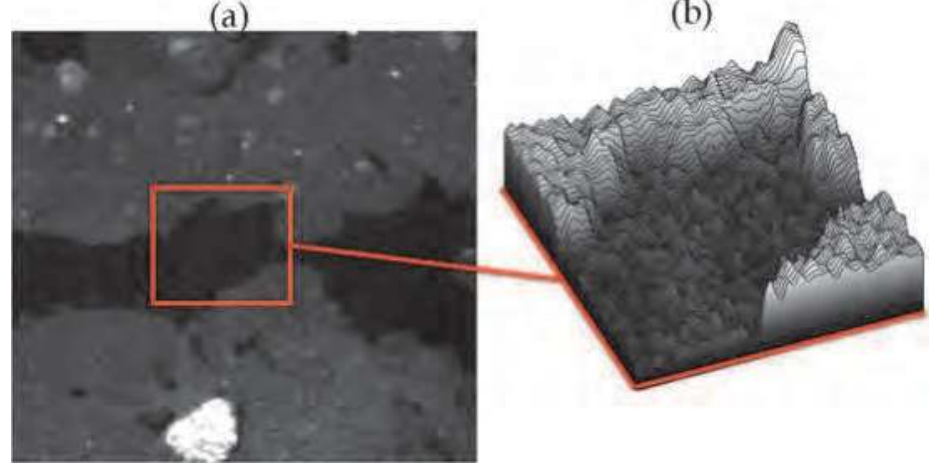

Fig. 2. An example of a noisy $\mathrm{CX} \mu \mathrm{T}$ image (a) and the intensity profile plot for the area marked on the $\mathrm{CX} \mu \mathrm{T}$ image (b).

In addition to ESF, the tomographic reconstructions are often contaminated by imaging noise. Collecting photons with Charge-Coupled Device (CCD) is a time-dependent discrete procedure that presents Poisson noise into the collected data. Utilisation of an analogue-todigital -converter such as in CCD causes Gaussian -type of noise into the images. In the final $3 \mathrm{D}$ representation of the tomographic sample, noise can be seen as random variation of grey values. This effect causes edge blurring. Furthermore, when binarised by thresholding procedure, falsely labelled voxels can appear.

Many algorithms have been developed to decrease the noise, e.g. anisotropic diffusion (Perona \& Malik, 1987), bilateral filtering (Tomasi \& Manduchi, 1998) and SUSAN filtering (Smith \& Brady, 1997). However, none of the filtering methods is perfect and post processing is often necessary to reduce the artefacts from the binarised images.

\subsubsection{Image segmentation}

Segmentation means separation of the image into interesting sub-regions for further analyses. For segmentation of 3D reconstructions, there is a large variety of algorithms. To mention few the most commonly used, are global algorithms like thresholding, spatially aware algorithms like region grow and watershed (Gonzales \& Woods, 2002), optimisation based algorithms like level sets (Osher \&, Sethian, 1988) or active contours (Kass et al, 1987), shape -based methods like LOG or DOG (Gonzales \& Woods, 2002), texture -based methods like local binary patterns (Ojala et al, 1994), and combinations of these. Often the complexity of the required algorithm is directly proportional to the signal-to-noise ratio of the original image.

A typical task in segmentation procedure is to separate the image of a porous media to solid and void phase. In optimal conditions, the phases can be distinguished by the difference in their grey value distributions. However, the grey value distributions are often overlapping due to imaging noise. In many cases, thresholding combined with post processing gives satisfactory results. The post processing procedures aim to remove the non-connected parts, i.e. the "levitating" solid objects from the images or to fill the small non-effective (isolated) pores. In the cases where the overlapping regions of the grey value distributions are wide, more sophisticated algorithms are needed. 
We have developed a so-called forest fire algorithm to separate the material phases when their grey value distributions are overlapping too much for simpler methods. As an input, a user will give the limits for the overlapping area in the grey value histogram. The algorithm processes the grey values in between the given limits and decides whether it is solid or void by adding more voxels to each phase iteratively. In addition to grey value information, spatial information is incorporated. The voxels are added into the group if there are enough members of the same group around it. By adjusting the number of required neighbours, the sensitivity of the method can be adjusted individually for each phase. In practice, the method is closely related to region grow method, but it enhances the traditional region grow by adding a weak "surface tension" to it. The benefits of the method are smooth surfaces and possibility to alter the volume of selected phase by allowing either solid or void to conquer its area easier. The disadvantage of the method is that some of the smallest details can be lost. The name forest fire comes from simple forest fire simulations where forest is divided into cells which will catch fire if certain amounts of its neighbouring cells are already burning.

\section{Fluid flow permeability analysis}

Permeability $\kappa$ is a tensor valued measure of the ability of a porous material to transmit fluids. It is defined for slow, steady-state, isothermal, Newtonian fluid flow through a porous medium by Darcys's law (Darcy, 1856; Bear, 1972)

$$
\vec{q}=-\frac{1}{\mu} \kappa \times \tilde{N} \psi
$$

where $\vec{q}$ is the superficial volume flux vector and $\mu$ is the dynamic viscosity of the fluid. The pietsometric head $\psi$ is defined by equation $\nabla \psi=\nabla p-\rho \vec{g}$ where $p$ is the pressure and $\rho$ is the density of the fluid, and $\vec{g}$ is the acceleration due to a body force. In general, permeability is a symmetric second-order tensor (Liakopoulos, 1965):

$$
\kappa=\left[\begin{array}{lll}
k_{x x} & k_{x y} & k_{x z} \\
k_{y x} & k_{y y} & k_{y z} \\
k_{z x} & k_{z y} & k_{z z}
\end{array}\right]
$$

Several theoretical results for permeability coefficients have been reported in the literature. Perhaps the most common formula which can be derived analytically for simplified capillary model is the Kozeny-Carman relation

$$
k=\frac{1}{c \tau^{2} S_{0}^{2}} \frac{\phi^{3}}{(1-\phi)^{2}}
$$


where $c$ is a constant that depends on the cross section of the capillaries (being $c=2$ for circular cross section), $s_{0}$ is the specific surface area of the sample, $\tau$ is the tortuosity of the flow and $\phi$ is the porosity of the media (Bear, 1972; Dullien, 1979).

\subsection{Numerical method}

Numerical permeability analyses were done directly on the voxel model of the samples. The diagonal elements of permeability tensor $\kappa$ (in Eq. (2)) were obtained within FDM by first solving over a periodic REV the following boundary value problem arising from the homogenisation process:

$$
\begin{array}{ll}
\mu \nabla^{2} \vec{v}-\nabla \delta p-\nabla \psi=0, & \text { in } \Omega_{f} \\
\nabla \cdot \vec{v}=0 & \text { in } \Omega_{f}, \\
\vec{v}=0 & \text { on } \Gamma
\end{array}
$$

where $\Omega_{f}$ and $\Gamma$ represent the fluid volume and the fluid-solid interface, respectively. These three equations are the conservation of momentum, conservation of mass and the noslip condition on the fluid-solid interface. Here $\vec{v}$ stands for the periodic microscopic velocity field, $\nabla \psi$ is the pietsometric head and $\delta p$ is the first order periodic fluctuation of the pressure $p$.

The boundary value problem Eq. (4) was solved by using FDM implemented in GeoDict2010 R1 (64 bit Linux) software. In the method, the velocity $\vec{v}$ and the pressure $p$ are discretised on a staggered grid: velocities are defined on their respective voxel faces and the pressure is defined in the centre of the voxel. Then the partial differential equations are solved by using the FFF-Stokes solver based on Fast Fourier Transform. This solver appears to be fast and memory efficient for large computations dedicated to 3D images (Wiegmann, 2007). Finally, the permeability coefficients of the samples can be deduced from equations (1) and (2) by calculating the volume average of the velocity field over the REV $(\langle\vec{v}\rangle=\vec{q})$ for a given macroscopic gradient of pressure $\nabla \psi$.

During the permeability analysis, periodic boundary condition was enforced on all the sample faces. For tomographic samples, ten extra fluid layers were added in flow direction on both sides of the sample volume. This was done in order to mimic the experimental measurement conditions, see Koivu et al., (2009a) and Koivu et al., (2009b).

\subsection{Experimental method}

Permeability coefficients $k_{z z}$ of the samples were measured experimentally using the permeability measurement device (PMD) presented in refs (Koivu et al., 2009a; Koivu et al., $2009 \mathrm{~b})$. The PMD can be utilised for measuring permeability of porous materials using both liquids and gases as permeating fluids.

For the purpose of this work, the PMD was modified such that the sample was compressed only at its peripheral part to prevent any flow on that region, and the central part was left fully open for flow; see a schematic illustration of the measurement set-up in Fig. 3a. The measured sample size for the wool fibre web and the packaging board had $90 \mathrm{~mm}$ diameter. For the measurements, the sand stone samples with diameter of $35 \mathrm{~mm}$ and thickness 10 $\mathrm{mm}$ was attached with silicone glue into a special sample holder, see Fig. 3b. 

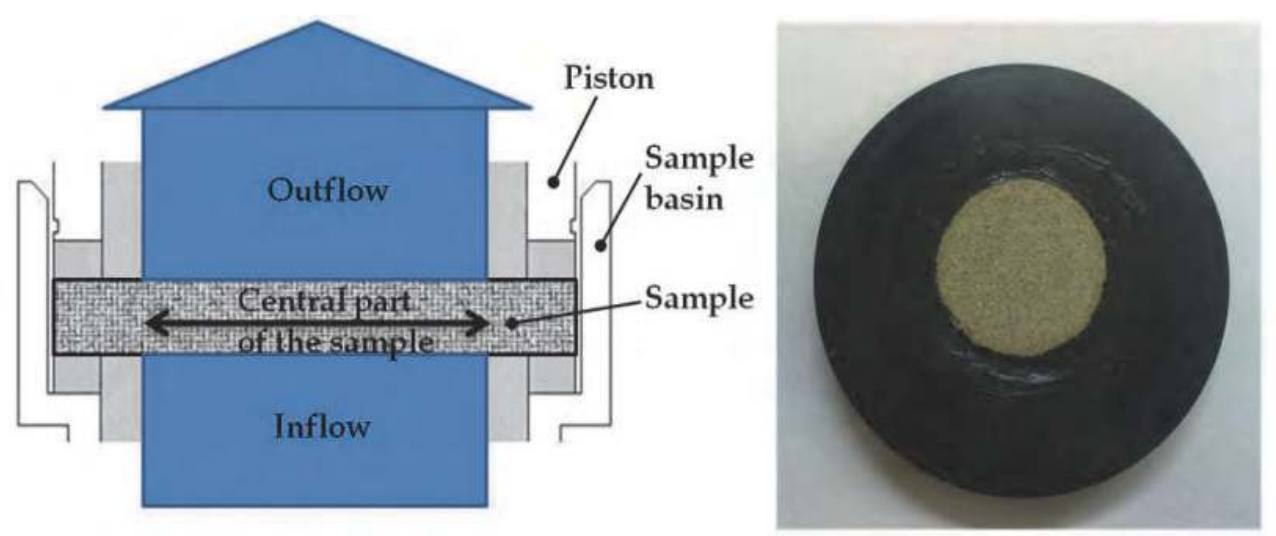

Fig. 3. Schematic illustration of the measurement set-up (a) and a sandstone sample attached in the sample holder (b).

In the experimental method, the diagonal values of the permeability tensor in the case dependent coordinate system (see e.g. Fig. 1) are found using the integrated form of Darcy's law

$$
k_{i i}=-\frac{\mu q_{i}}{\Delta P / \Delta L_{i}}=\frac{\mu Q \Delta L_{i}}{A\left(P_{\text {out }}-P_{\text {in }}\right)}
$$

where $Q$ is the volumetric flow rate through the sample, $A$ is the cross-sectional flow area, $\Delta P=P_{\text {out }}-P_{\text {in }}$ is the pressure drop and $\Delta L_{i}$ is the length over which the pressure drop takes place.

Experiments were conducted with air flow in order to prevent structural changes due to swelling of sample material. This is important in order to obtain similar structure of materials in experiments and in numerical flow solution based on the pore geometry given by tomographic images of dry material samples. Equation (5) is valid for the incompressible fluid flows. For gas flows through porous medium, Darcy's law must be slightly modified to account for compressibility effects. For isothermal compressible flow the permeability coefficient is thus given by

$$
k_{\text {ii }}=-\frac{\mu q_{i}}{\Delta P / \Delta L_{i}} \frac{P_{\text {out }}}{P_{\text {ave }}}=\frac{\mu Q \Delta L_{i}}{A\left(P_{\text {out }}-P_{\text {in }}\right)} \frac{P_{\text {out }}}{P_{\text {ave }}}
$$

where $P_{\text {out }}$ is the pressure, $Q$ is the volumetric flow rate at the downstream side of the medium, and $P_{\text {ave }}=P_{\text {out }}+1 / 2\left(P_{\text {in }}-P_{\text {out }}\right)$ (Bear, 1972; Leskelä \& Simula, 1998).

In the experimental approach, the values of the permeability coefficient in z-direction $\left(k_{z z}\right)$ were calculated using Darcy's law for compressible fluid flow by Eq. (6). The coordinate conventions for the sample types are shown in Fig. 1. Measurements were repeated for five macroscopically identical samples in order to obtain an estimate of the statistical uncertainty of the results. The statistical uncertainty of the experimental results was $20 \%$. 


\section{Results}

We proceeded in two steps. First, we used a numerical method to find values of permeability for fibrous porous media based on regular hexagonal array of cylinders. The numerical results thus obtained were compared with the analytical results found in the literature. In the second step, we used the numerical method to find values of permeability coefficient for the wool fibre web, packaging board and sandstone samples. These results were compared with the experimental results obtained by using the PMD (Koivu et al., 2009a; Koivu et al., 2009b).

\subsection{Regular arrays}

Artificial sample geometries of hexagonal arrays of cylinders were prepared to test and demonstrate the effect of different imaging artefacts on structure and numerical fluid flow analysis. The volume size of the simulation geometries were $693 \times 400 \times 100$ pixels and the porosity of the cylinder arrays was selected to be $50 \%$.

\subsubsection{Effect of noise}

Visualisations of flow speed field of four hexagonal arrays of cylinders having different levels of noise are shown in Fig. 4. The noise levels are defined as percentage of the faulty voxels of the total volume of the sample.

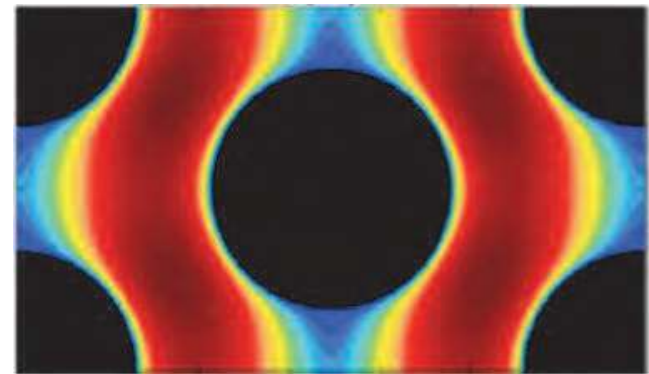

(a)

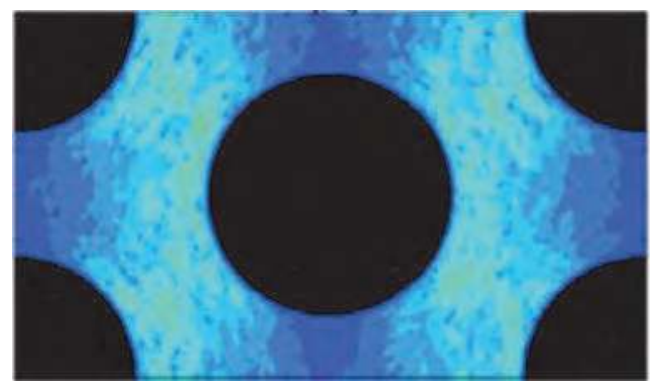

(c)

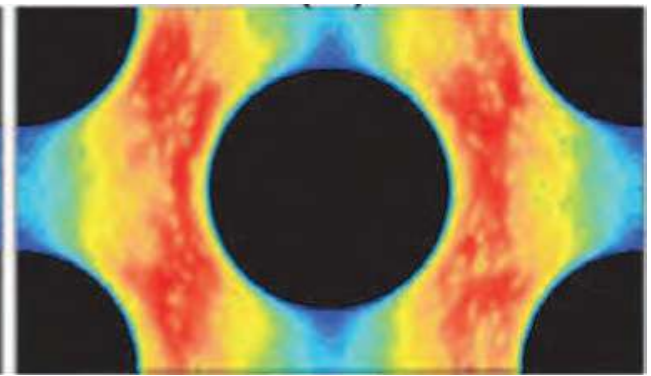

(b)

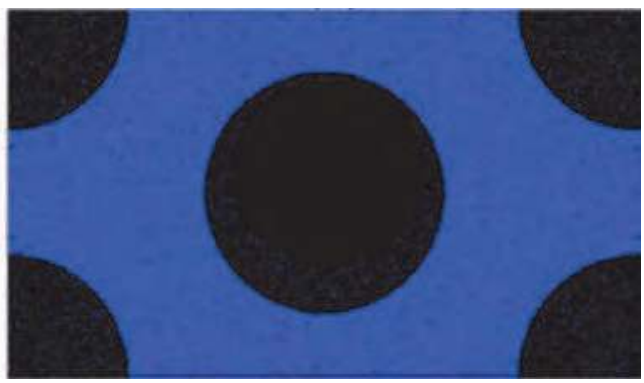

(d)

Fig. 4. Visualisation of flow speed field of four hexagonal arrays of cylinders having different levels of noise: (a) $0 \%$, (b) $0.05 \%$, (c) $0.3 \%$ and (d) $5 \%$. Flow direction is in these cases from top to bottom. Red and yellow colours represent high flow speed and green and blue low flow speed. 
Numerically analysed permeability values and corresponding (noise free) analytical value by Drummont \& Tahir (1984) are shown in Fig. 5. According to the results, even a small amount of noise has a drastic influence on fluid flow permeability.

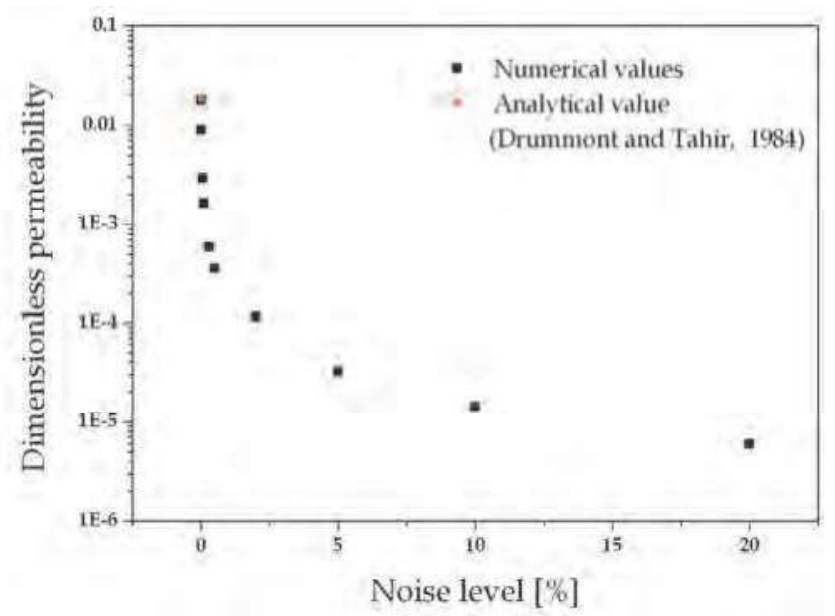

Fig. 5. Numerically solved permeability values for the hexagonal array of cylinders as a function of noise level. For comparison, analytical value for noise free geometry is also given (Drummont \& Tahir, 1984).

The effect of noise on specific surface area was evaluated as a function of noise level, see Fig. 6 . The specific surface areas were analysed utilising the marching cubes algorithm (Lorensen \& Clive, 1987a; Lorensen \& Clive, 1987b). Increase in the amount of noise increases the specific surface area of the simulation geometry and thus decreases the permeability value, see Eq. (3).

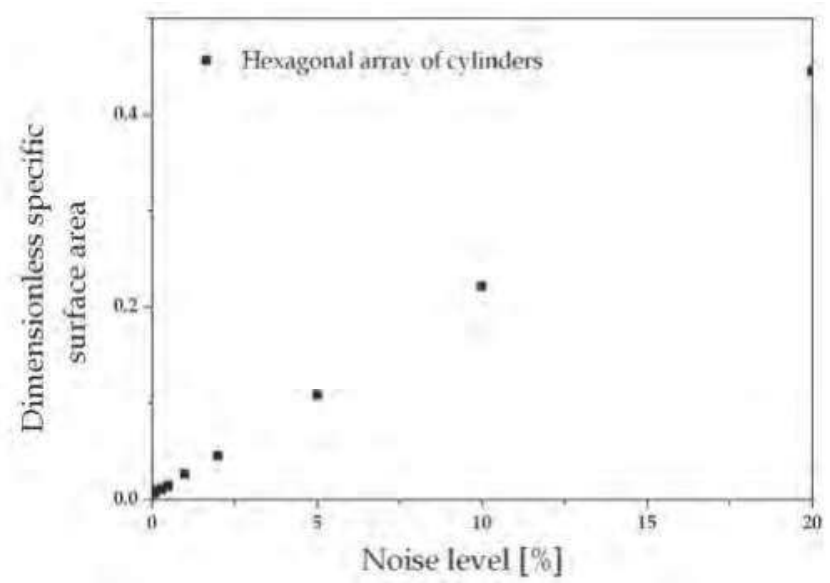

Fig. 6. Dimensionless specific surface area as a function of noise level for the hexagonal array of cylinders. 
The effect of noise on pore size distribution was evaluated as a function of noise level, see Fig. 7. The pore size distributions were determined with the so-called sphere fitting algorithm. In the sphere fitting method, the pore space is filled by non-overlapping spheres. The distribution of the radii gives estimation for the pore size distribution (Wu et al., 2007). The pore size distribution was found to change dramatically as a function of noise level. The mode value of the distribution of the geometry with the noise level of $0.01 \%$ was approximately one third of the mode value for the noise free geometry.

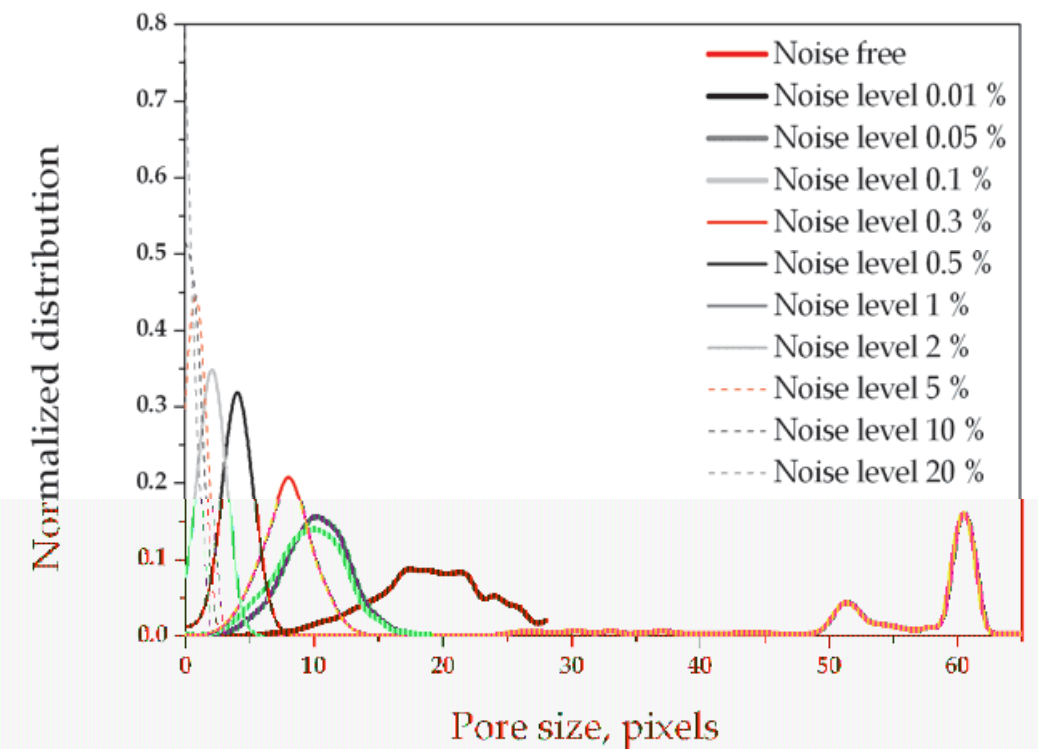

Fig. 7. Normalized pore size distribution for the hexagonal array of cylinders as a function of pore size in pixels with different noise levels.

\subsubsection{Effect of edge blurring}

Grey scale profiles on solid-void boundaries of a material in tomographic images are often blurred due to ESF. Imaging noise combined with blurred boundaries can cause the thresholded solid-void boundary of the final reconstruction to look rough, see visualisation in Fig. 8b. Without noise, the pure edge spreading causes changes mainly in the porosity value of the sample geometry and thus changes in the permeability value, see e.g. the results published by Koivu et al. (2010). When noise is incorporated, the surface roughness will have an effect to the fluid flow close to the surfaces.

Artificial edge roughness was generated into the geometries of the hexagonal arrays of cylinders, see visualisations of the rough edges in Fig. 8a. To generate the simulation geometries the original geometry was blurred using standard 3D Gaussian blur. Gaussian distributed noise with known standard deviation was added to the blurred image. The image was then thresholded using 128 as the threshold value (in the original image void is 0 and solid is 256). Finally, all the particles not touching the solid phase were removed. The number of the edge roughness level corresponds to the standard deviation of the Gaussian distributed noise. 


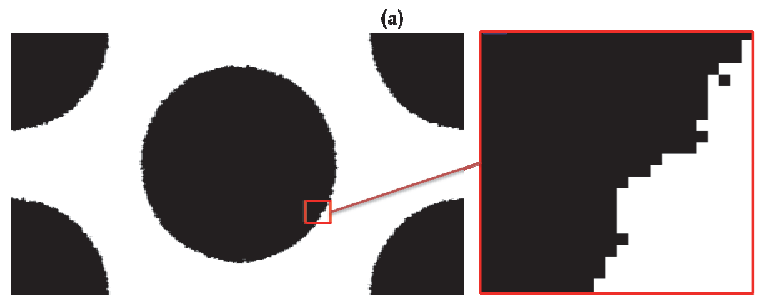

(b)

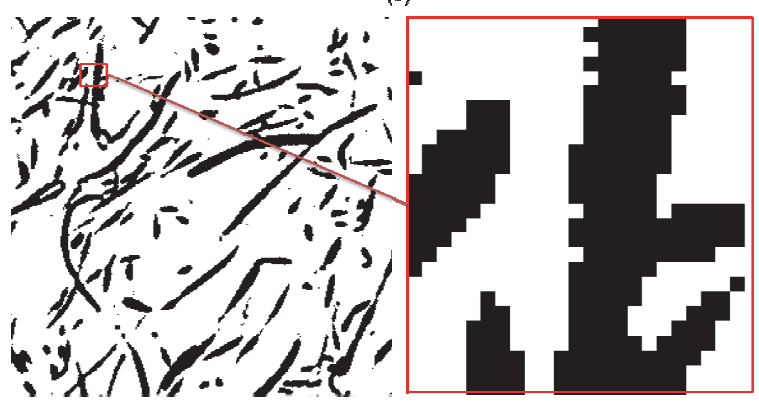

Fig. 8. Visualisations of artificially generated edge roughness on the hexagonal array of cylinders (a) and edge roughness on reconstruction of wool fibre web caused by $\mathrm{CX} \mu \mathrm{T}$ and image processing methods (b).

Edge roughness on solid-void boundary increases the specific surface area of the sample and therefore decreases the permeability value, see Figs 9 and 10. Edge roughness has only small effect on the pore size distribution, see Fig. 11.

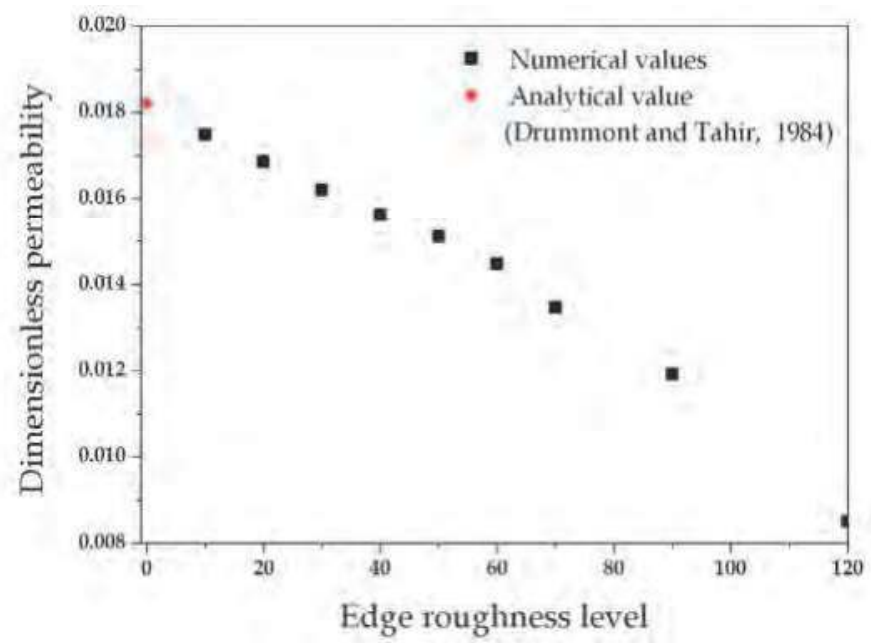

Fig. 9. Numerically solved permeability values for the hexagonal array of cylinders as a function of edge roughness level. Analytical value for noise free geometry is also given (Drummont \& Tahir, 1984). 


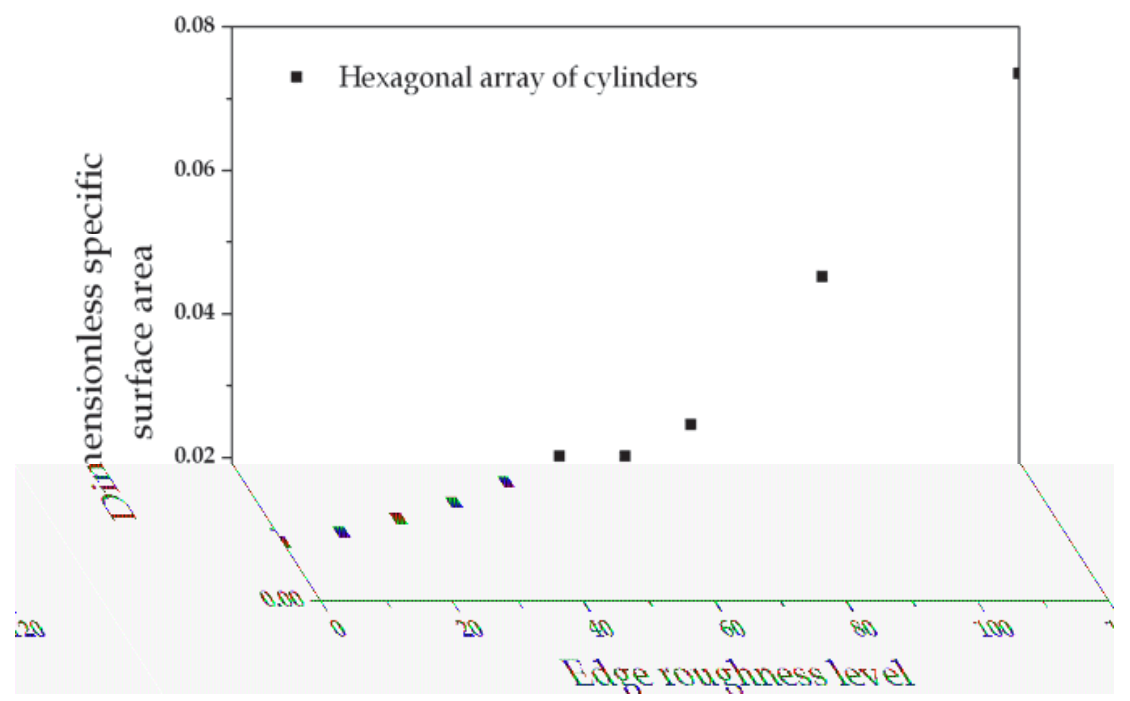

Fig. 10. Dimensionless specific surface area as a function of edge roughness level for the hexagonal array of cylinders.

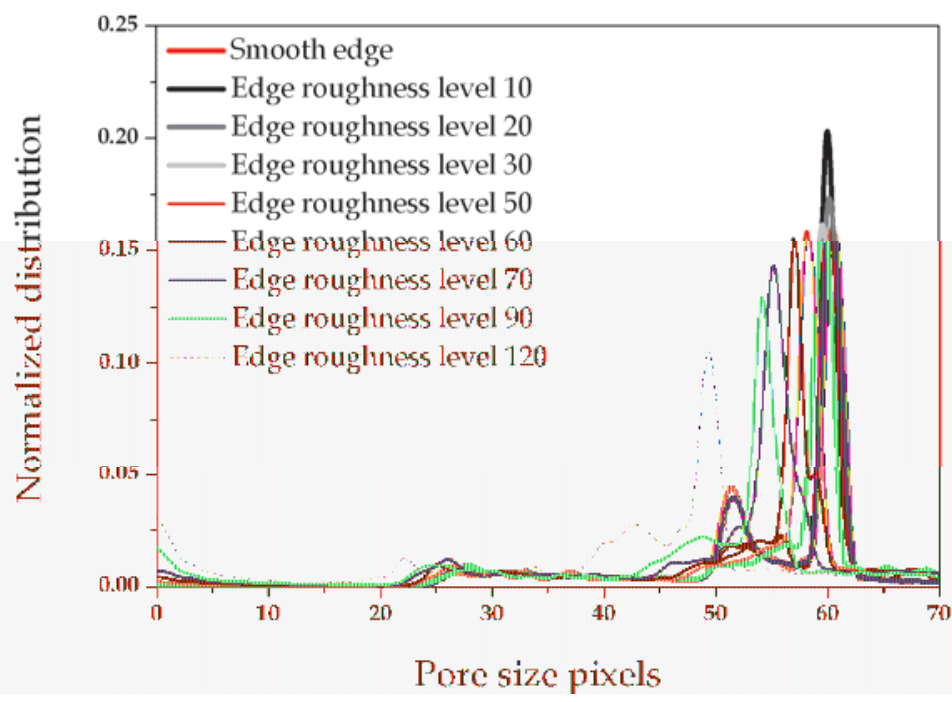

Fig. 11. Normalized pore size distribution as a function of pore size in pixels for the hexagonal array of cylinders with different edge roughness levels.

\subsection{Tomographic geometries}

Tomographic sample geometries were obtained with three different imaging methods. This was done in order to demonstrate the effects of very common imaging artefacts, which are produced by most of the tomographic imaging methods. 
The Effect of Tomography Imaging Artefacts on

\subsubsection{Effect of artefacts on fluid flow permeability}

Visualisations of wool fibre web sample with different threshold values are shown in Fig. 12. The sample geometries in (a) - (c) were denoised and then binarised using grey value based threshold (Gonzales \& Woods, 2002). At the lowest threshold levels (a) and (b), the noise is clearly visible in the void space. While threshold value was gradually increased, the noise became less evident and the thickness of the fibres (or size of the solid particles) diminished. The sample geometry in Fig. 12d was segmented utilising the forest fire method. The forest fire method was found to give noise free pore space and a fibre radius that corresponded well with the mean value obtained from scanning electron microscope images, $20 \mu \mathrm{m}$.

(a)

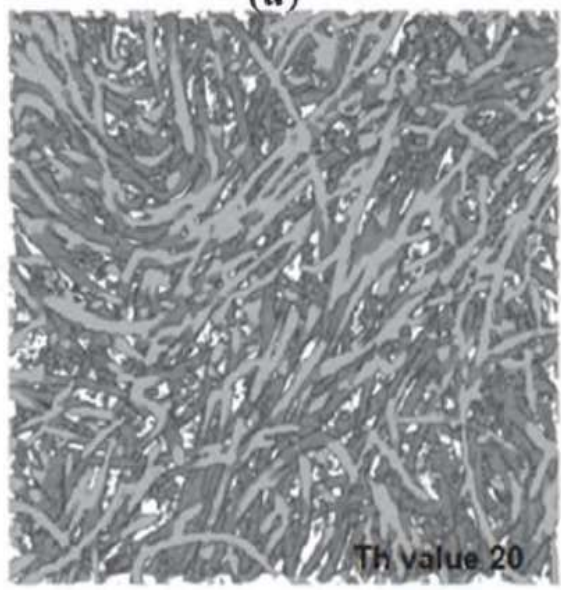

(c)

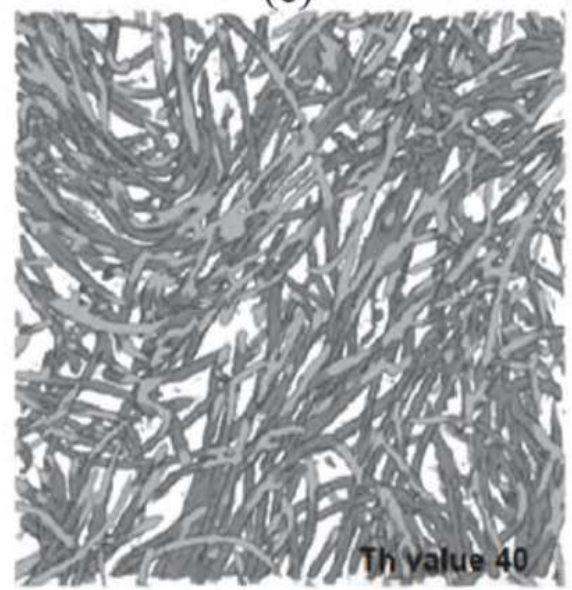

(b)

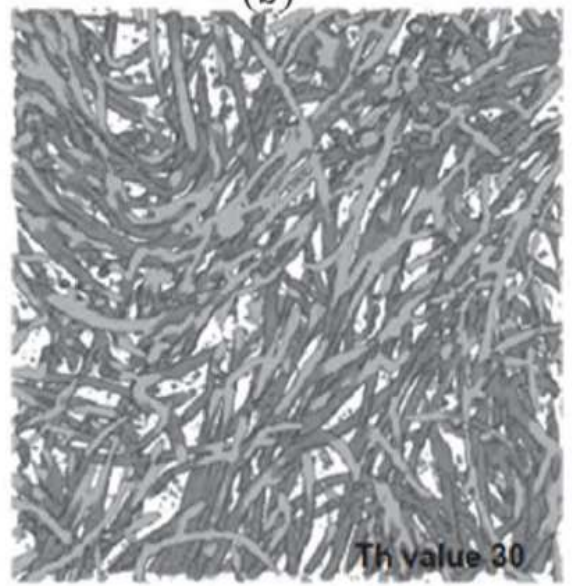

(d)

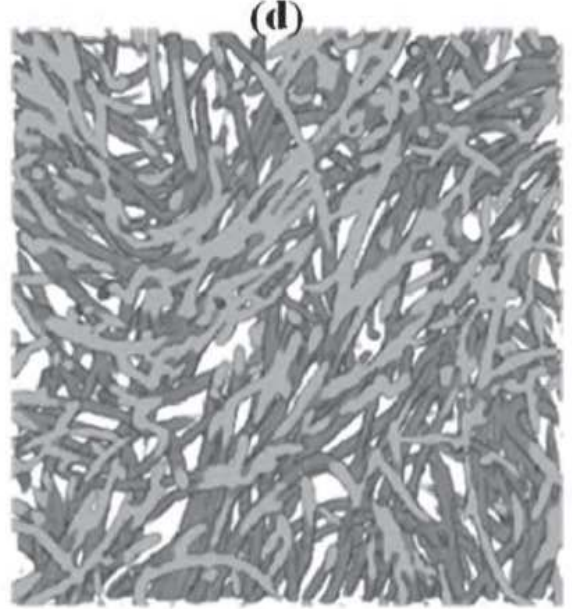

Fig. 12. Visualisation of segmented wool fibre web sample at different threshold values: (a) 20, (b) 30 and (c) 40, and with the forest fire method (d). 
Grey value -based thresholding procedure with different threshold values were conducted for all the three tomographic sample types. Numerical fluid flow permeability analyses were conducted for all the geometries and the obtained values were compared with experimental results, see Fig. 13.

As an example, for the wool fibre web, the "optimal" threshold value would have been between 20 and 30, see Fig. 13a. By visually examining the corresponding images, it was found that there was still noise left in flow channels at threshold value 30. Permeability at the optimal threshold value would not have been result of the realistic sample geometry, but combination of noise causing higher specific surface area and thinner fibres increasing the porosity of the geometry. The results were found qualitatively similar for the packaging board and the sand stone samples.

The forest fire method was found to be efficient tool for segmentation procedure. Numerical permeability values for the geometries segmented by the forest fire method were $2.5 \mathrm{E}-11 \mathrm{~m}^{2}$, $4.8 \mathrm{E}-14 \mathrm{~m}^{2}$ and $2.5 \mathrm{E}-12 \mathrm{~m}^{2}$ for the wool fibre web, the packaging board and the sand stone samples, respectively. Corresponding experimental measurement results for the samples were $2.5 \mathrm{E}-11 \mathrm{~m}^{2}, 5.1 \mathrm{E}-14 \mathrm{~m}^{2}$ and $2.5 \mathrm{E}-12 \mathrm{~m}^{2}$. The maximum difference between the experimental results and the numerical permeability values found by using the forest fire segmented sample geometries was in these cases less than $6.5 \%$. The statistical uncertainty of the experimental results was $20 \%$.

The numerical permeability results for the samples with different added noise levels (proportion of false voxels) are shown in Fig. 14. Artificial noise levels were generated into the sample geometries which were segmented by the forest fire method. The effect of noise on permeability was found to be qualitatively similar as for the hexagonal cylinder array. Increase in noise level caused decrease in fluid flow permeability value.

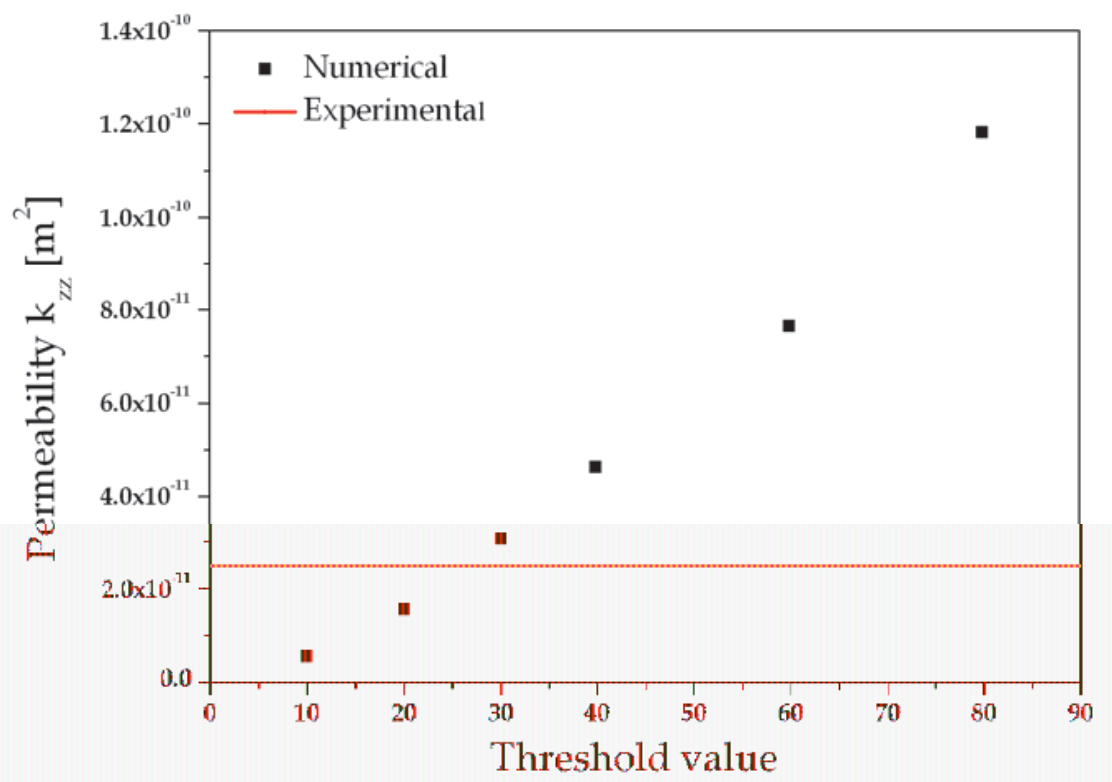

Fig. 13. (a) 


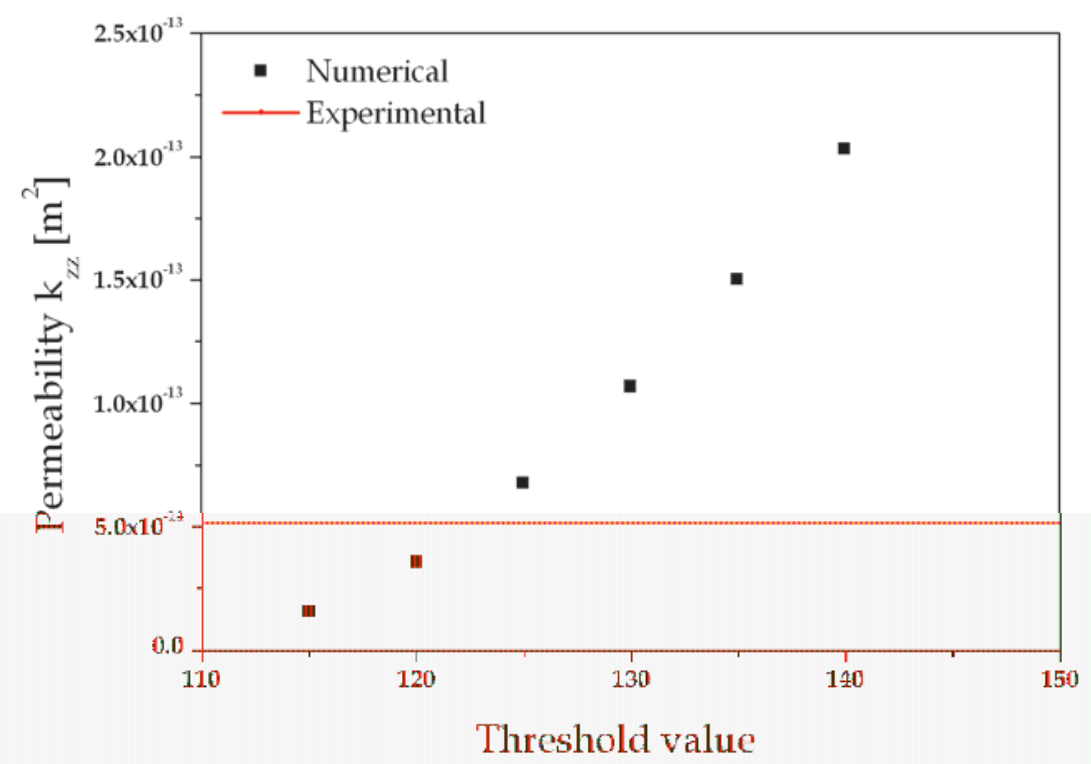

Fig. 13. (b)

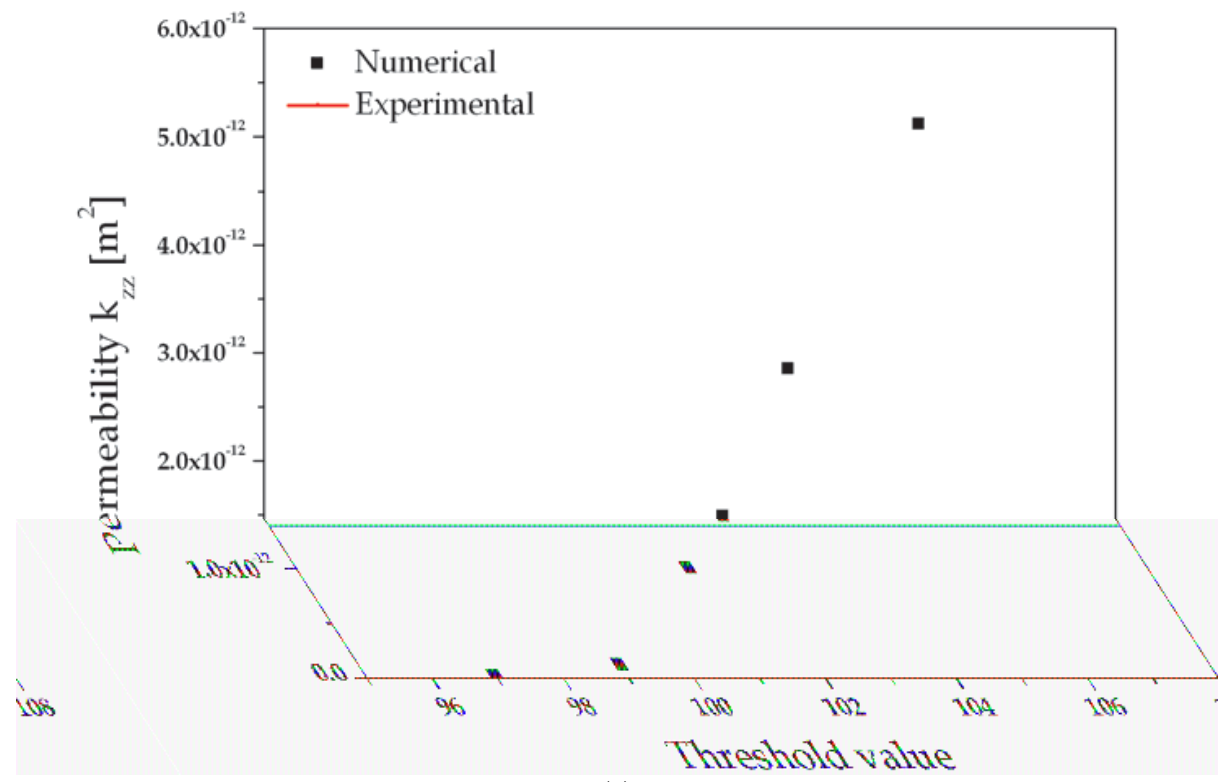

(c)

Fig. 13. Numerically solved permeability coefficients for the wool fibre web (a), the packaging board (b) and the sand stone (c) as a function of threshold value. Experimental results are given as an average of the five measurements for each sample types. 


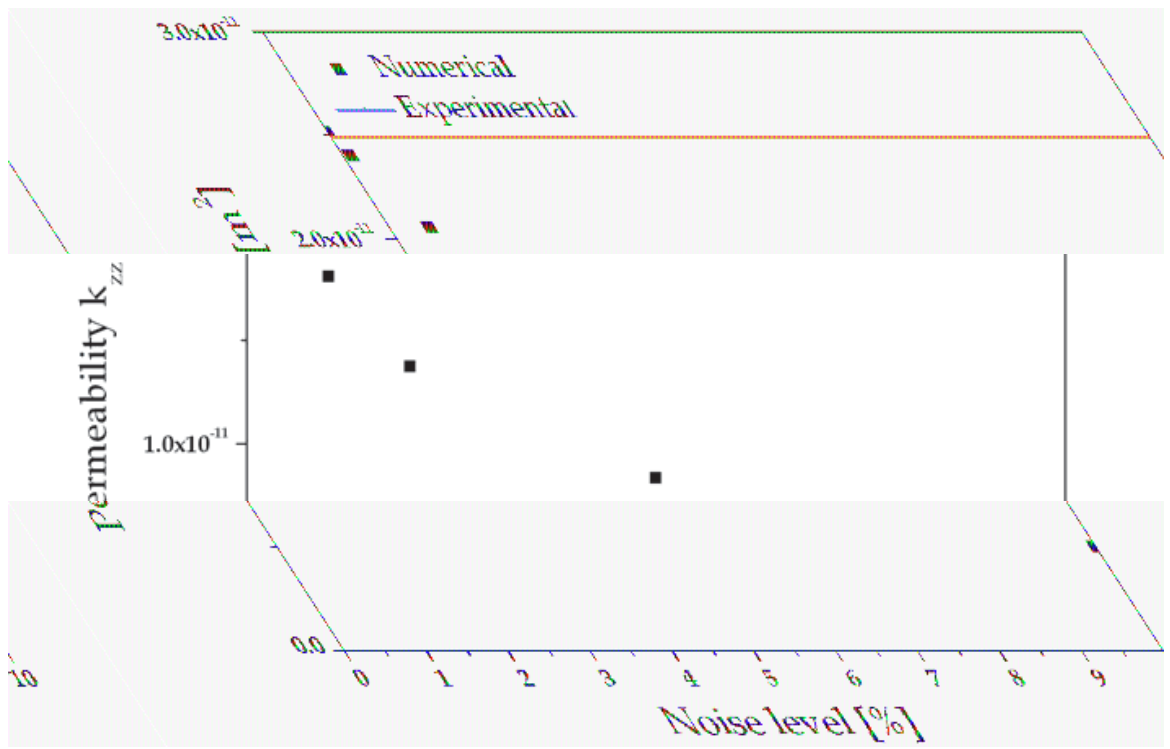

Fig. 14. (a)

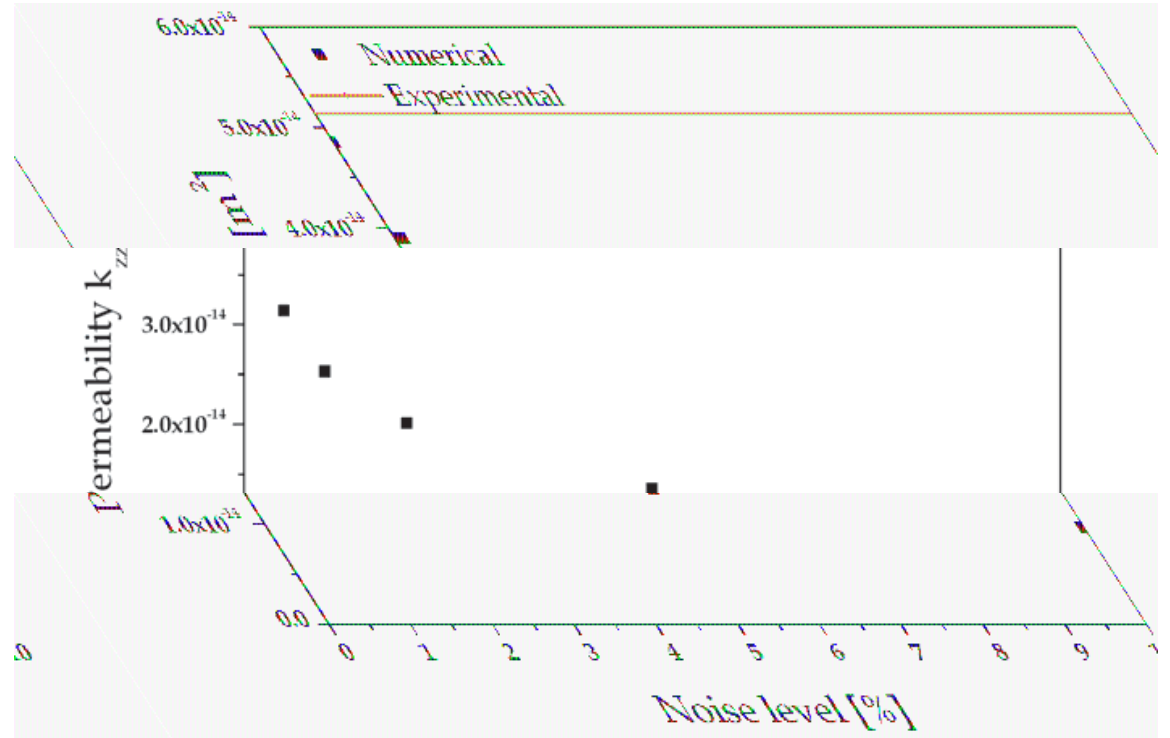

Fig. 14. (b) 


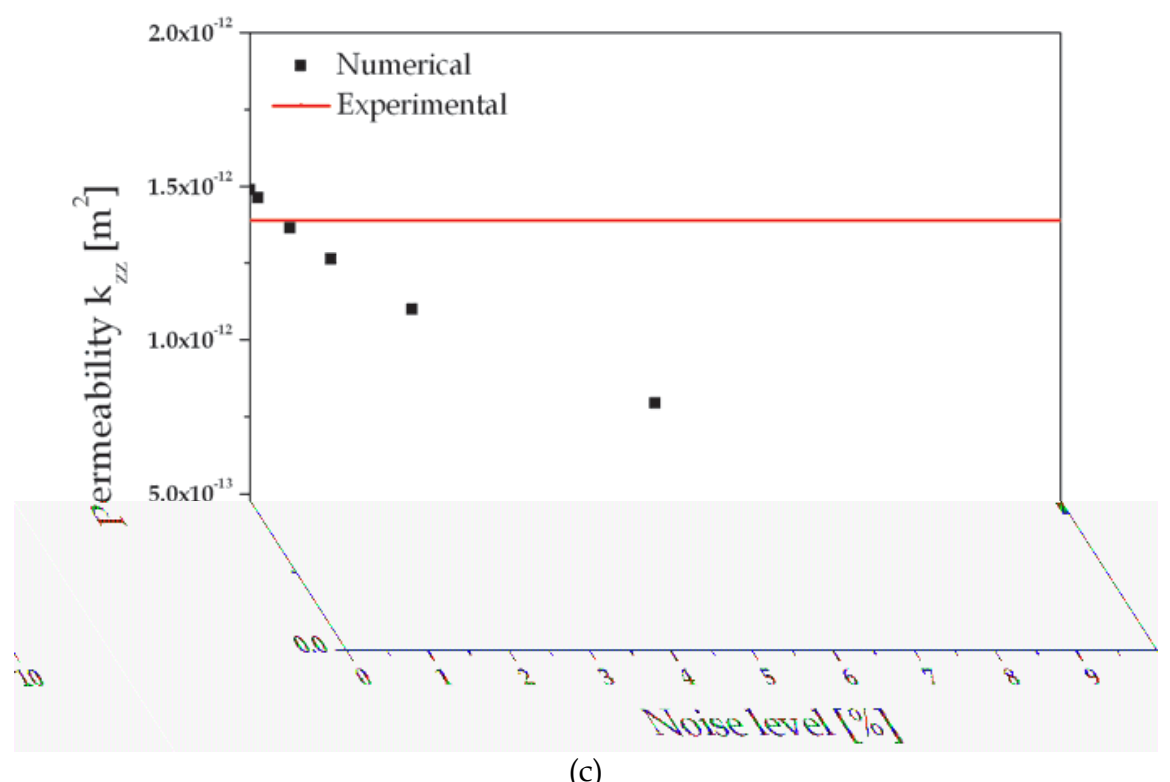

(c)

Fig. 14. Numerically solved permeability coefficients for the wool fibre web (a), the packaging board (b) and the sand stone (c) as a function of noise level. Experimental results are given as an average of the five measurements for each sample types.

\subsubsection{Effect of artefacts on specific surface area and pore size distribution}

Specific surface area of the wool fibre web sample was analysed as a function of threshold value and noise level, see the results in Fig. 15. The specific surface area of the geometry thresholded by the forest fire method had a value of $0.175 \mathrm{~m}^{-1}$. The value of specific surface area of the sample geometry decreased as a function of increasing threshold value and increased as a function of increasing noise level. According to Eq. (3), when a sample geometry has a high specific surface area, it has a small permeability value. This could also be seen from the results in Fig. 15 and in Figs 13a and 14a.

Pore size distribution of the wool fibre web sample geometry was analysed as a function of threshold value, see the results in Fig. 16a. When compared to the experimental permeability results, the optimal threshold value was found to be between 20 and 30, see Fig. 13a. The results in Fig. 16a clearly show that with the threshold values of 20 or 30 the pore size distribution differs highly from the pore size distribution of the geometry that was segmented using the forest fire method. These results support the interpretation related to the permeability values obtained using the geometries segmented by conventional grey value -based thresholding. Combination of too high specific surface area and too thin fibres (high porosity) resulted to the permeability values very close to the experimental results. These interpretations were found to be similar for the packaging board and for the sand stone samples also.

Pore size distribution as a function of noise level for the wool fibre web sample is presented in Fig. 16b. The effect of added noise was found to be not as high as for the hexagonal array of cylinders, see Fig. 7. For noise level of $2 \%$, the mode value of the distribution was approximately a half of the mode value of the noise free geometry. 


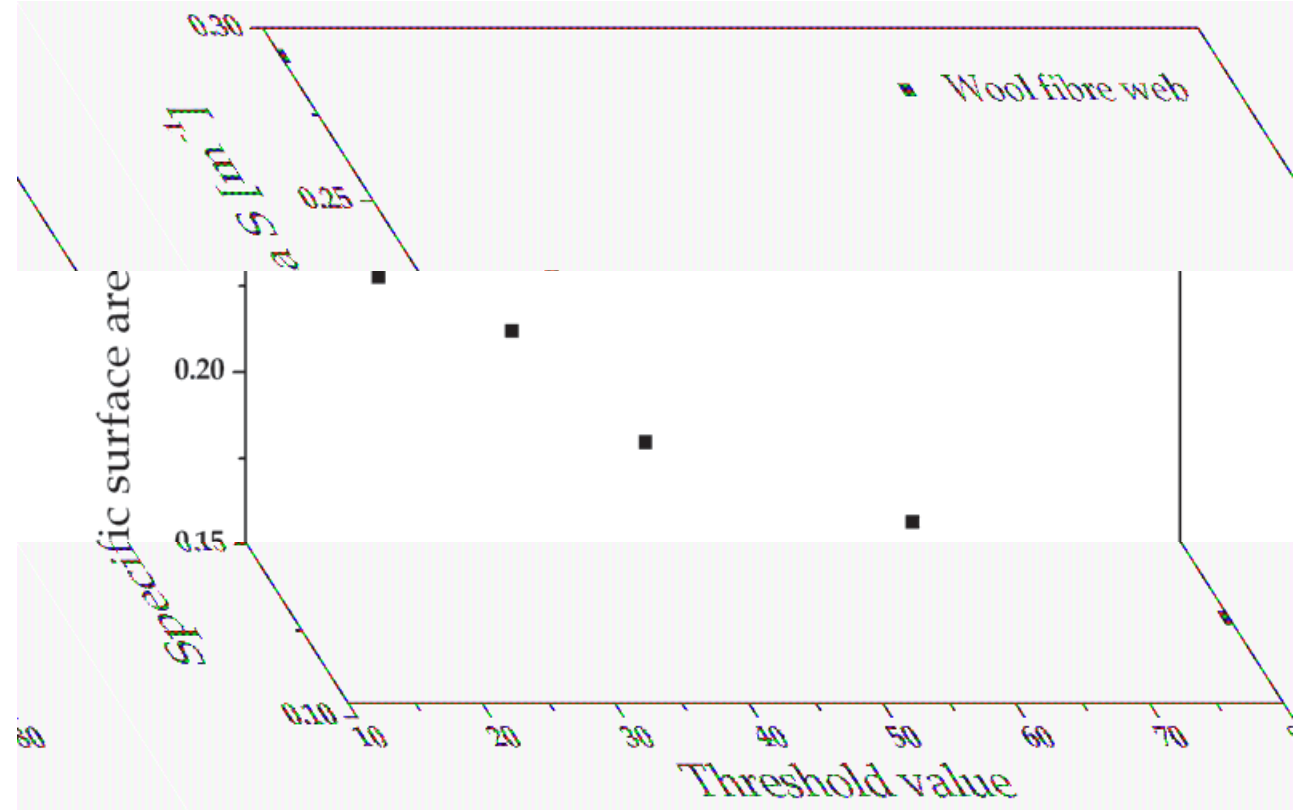

Fig. 15. (a)

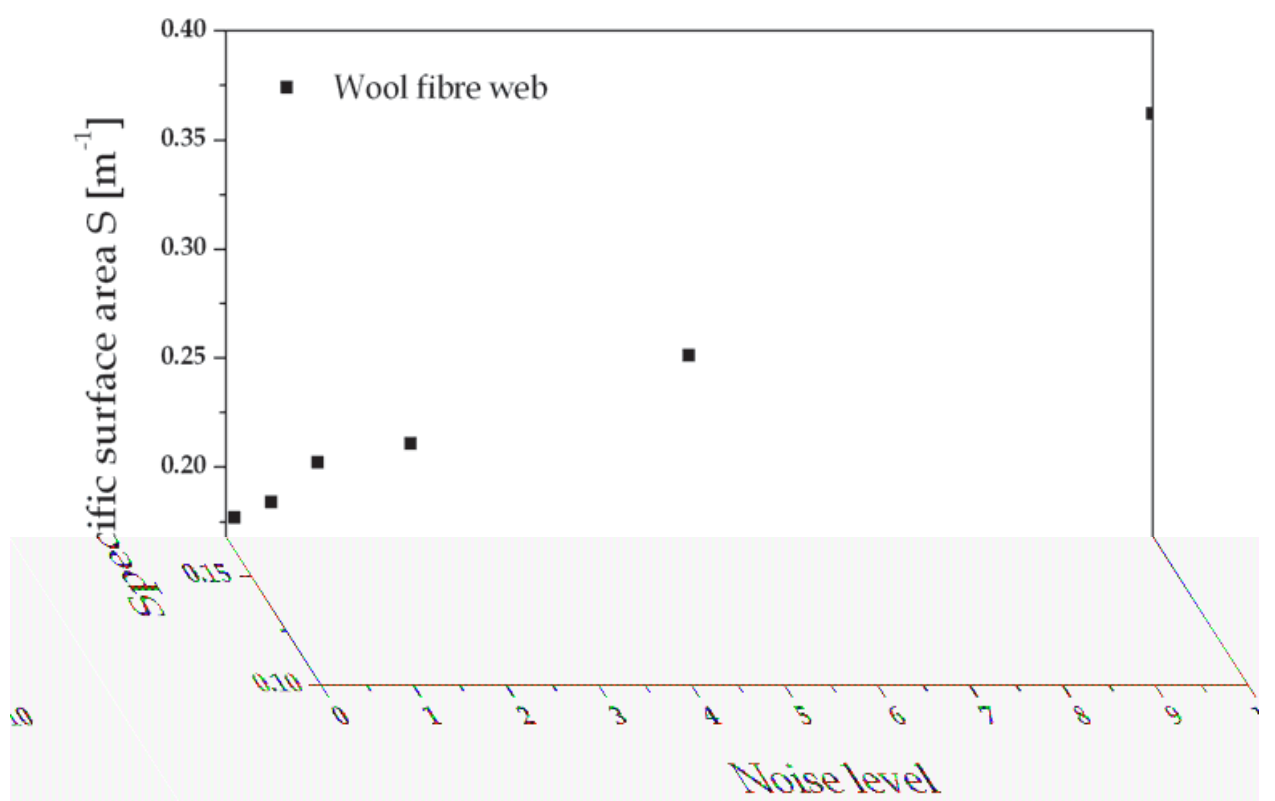

(b)

Fig. 15. Specific surface area of the wool fibre web sample as a function of threshold value (a) and noise level (b). 


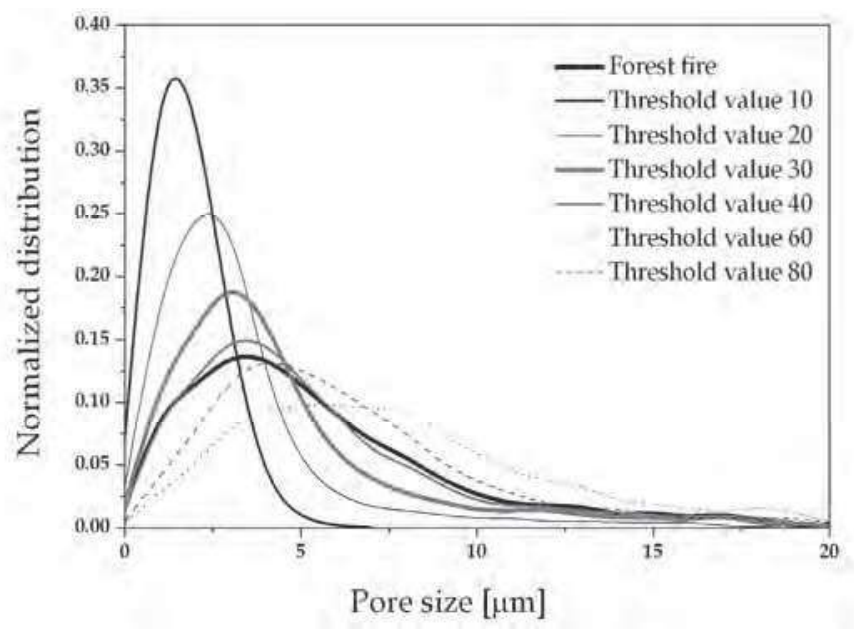

Fig. 16. (a)

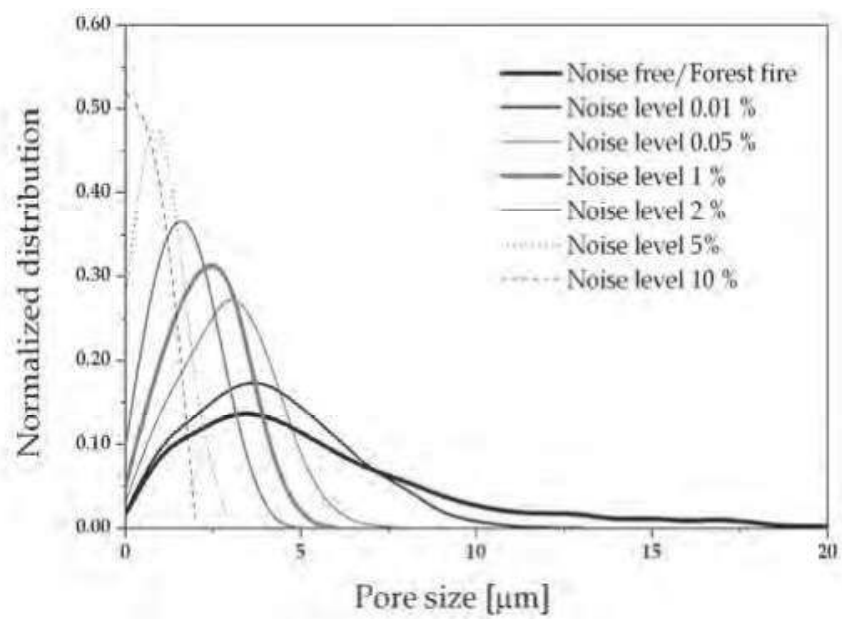

(b)

Fig. 16. Normalized pore size distribution for the wool fibre web sample as a function of pore size with different threshold values (a) and with different noise levels (b).

\section{Conclusions}

Approximate factors of how certain levels of noise and edge roughness effect on permeability value, mode value of pore size distribution and specific surface area of the hexagonal array of cylinders are summarised in Table 1. According to the results, even a small amount $(0.01 \%$ of sample volume) of noise in the void space has a drastic influence on the permeability values. The noise level of few percent caused magnitudes decrease in the permeability values. Increase in the amount of noise increases the specific surface area of the simulation geometry and thus decreases the permeability value. Also the pore size 
distributions of the simulation geometries were found to change dramatically as a function of noise level. The mode value of the pore size distribution with the noise level of $0.01 \%$ is approximately one third of the mode value of the noise free geometry.

Edge roughness on solid-void boundaries increases the specific surface area of the hexagonal cylinder geometry and therefore decreases the flow permeability value. The effect is, however, moderate compared to the effect of noise. Edge roughness was found to have only a small effect on the pore size distribution of the geometry.

Approximate factors of how certain levels of threshold value and noise effect on permeability value, mode value of pore size distribution and specific surface area of the wool fibre web sample are given in Table 2. The factors are given as values compared to the analysis results based on the forest fire segmented geometry that gives values very close to the experimental results. The results obtained using the forest fire method were compared to the data obtained from the scanning electron microscope images. Agreement with the sizes of characteristic solid objects was found to be good. The numerical permeability analyses for the sample geometries segmented using the forest fire method provided good agreement with the experimental results. The difference between the experimental results and the numerical permeability values found by using the forest fire segmented simulation geometries was less than $6.5 \%$ for all the sample types.

\begin{tabular}{|c|c|c|c|}
\hline & Noise level $0.01 \%$ & Noise level $1 \%$ & Noise level $10 \%$ \\
\hline Permeability & 0.5 & 0.01 & 0.001 \\
\hline $\begin{array}{l}\text { Mode value of pore } \\
\text { size distribution }\end{array}$ & 0.3 & 0.2 & 0.1 \\
\hline \multirow[t]{2}{*}{ Specific surface area } & 1.1 & 3 & 30 \\
\hline & $\begin{array}{c}\text { Edge roughness } \\
\text { level } 10\end{array}$ & $\begin{array}{c}\text { Edge roughness } \\
\text { level } 50\end{array}$ & $\begin{array}{c}\text { Edge roughness } \\
\text { level } 120\end{array}$ \\
\hline Permeability & 1 & 0.9 & 0.5 \\
\hline $\begin{array}{l}\text { Mode value of pore } \\
\text { size distribution }\end{array}$ & 1 & 0.9 & 0.8 \\
\hline Specific surface area & 1.2 & 2.5 & 9 \\
\hline
\end{tabular}

Table 1. Approximate factors of how certain levels of noise and edge roughness effect on permeability value, mode value of pore size distribution and specific surface area of the hexagonal array of cylinders. Factors are given as values compared to the results for the noise free geometry.

The permeability results were found to be very sensitive for the threshold value used in segmenting the 3D geometries. Faulty selection of the threshold value can cause an error for the permeability value by a factor of approximately 2-5. By using grey value -based threshold method, the permeability value might by chance have approximately correct values (compared to the experimental results). However, the permeability value with selected threshold value would not necessarily be result of the realistic sample geometry, but combination of noise causing higher specific surface area and too thin fibres increasing the porosity value of the geometry. This result was found to be qualitatively similar for all the three sample types and the imaging methods used here. 
Pore size distributions of the tomographic geometries were analysed as a function of threshold value. Although fluid flow analyses based on geometries segmented by grey value -based segmentation method might result in approximately correct permeability values, the structure of the pore geometry does not necessarily correspond with the realistic case. According to the results, the pore size distributions based on the grey value segmented geometries differ from the distributions based on geometries which were segmented using the forest fire method.

The forest fire segmented tomographic reconstructions were used in studying the effect of noise on tomographic geometries. Results in these cases indicate that the permeability results of tomographic geometries are not as sensitive to noise as in the case of regular hexagonal cylinder arrays. By increasing the noise level to $2 \%$, the permeability results decreased approximately by a factor of 0.5 .

The effect of noise level on pore size distribution was analysed. The effect of added noise on tomographic reconstructions is not as drastic as in the case of the hexagonal cylinder arrays. With the noise level of $2 \%$, the mode value of the distribution is approximately a half of the mode value for the noise free geometry.

The results indicate that effective methods for handling imaging artefact removal and segmentation are essential for obtaining reliable results for structural and transport properties by means of direct numerical analyses based on $\mathrm{CX} \mu \mathrm{T}$ reconstructions of the 3D structure of complex porous materials. According to our studies, the most essential factor for successful structural analysis and flow simulation is the quality of the tomographic reconstruction, i.e. noiseless and realistic structure. To achieve this requirement, a well controlled image acquisition and a good segmentation algorithm together with a verification based on a characteristic parameter, e.g. the mode value of pore size distribution measured by an independent method like mercury intrusion porosimetry or average characteristic dimension from a 2D microscopic image of the material, is required.

\begin{tabular}{|c|c|c|c|}
\hline & $\begin{array}{c}\text { Error in threshold } \\
\text { value }+10 \text { grey } \\
\text { values }\end{array}$ & $\begin{array}{c}\text { Error in threshold } \\
\text { value }+20 \text { grey } \\
\text { values }\end{array}$ & $\begin{array}{c}\text { Error in threshold } \\
\text { value + 30 grey } \\
\text { values }\end{array}$ \\
\hline Permeability & 2 & 3 & 5 \\
\hline $\begin{array}{c}\text { Mode value of pore } \\
\text { size distribution }\end{array}$ & 1.1 & 1.4 & 2 \\
\hline Specific surface area & 0.9 & 0.8 & 0.7 \\
\hline Noise level 2\% & Noise level 5\% & Noise level 10\% \\
\hline Permeability & 0.5 & 0.3 & 0.2 \\
\hline $\begin{array}{c}\text { Mode value of pore } \\
\text { size distribution }\end{array}$ & 0.5 & 0.2 & 0.1 \\
\hline Specific surface area & 1.2 & 1.2 & 2 \\
\hline
\end{tabular}

Table 2. Approximate factors of how certain levels of threshold value and noise effect on permeability values, mode value of pore size distribution and specific surface area of the wool fibre web. The factors are given as values compared to the analysis results based on the forest fire segmented geometry. 


\section{Acknowledgement}

The authors thank Dr. Keijo Mattila for fruitful discussions related to the project and M.Sc. Jarno Alaraudanjoki for the support related to the experimental permeability measurements. The authors express their gratitude to the ID19's team of ESRF for help with the acquisition of the images.

\section{References}

Aaltosalmi, U.; Kataja, M.; Koponen, A.; Timonen, J.; Goel, A.; Lee, G. \& Ramaswamy, S. (2004). Numerical analysis of fluid flow through fibrous porous materials, Journal of Pulp Paper Science, Vol.30, No.9, pp. 251-255

Bear, J. (1972). Dynamics of Fluid in Porous Media, Dover Publications inc., pp. 151-173

Belov, E. B.; Lomov, S. V.; Verpoest, I.; Peters, T.; Roose, D.; Parnas, R. S.; Hoes, K. \& Sol, H. (2004). Modelling of permeability of textile reinforcements: lattice boltzmann method, Composites science and techology, Vol64, pp. 1069-1080

Coles, M. E., D.; Hazlett, R., P.; Spanne, E.; Soll,W. \& Jones, E. L. K. W. (1998). Pore level imaging of fluid transport using synchrotron x-ray microtomography, Journal of Petroleum Science and Engineering, Vol.19, No.1, pp. 55-63

Darcy, H. (1856). Les fontaines publiques de la ville de dijon. Dalmont, Paris Drummont, M. I. \& Tahir, J. E. (1984). The permeability of fibrous porous media. International Journal of Multiphase Flow, Vol.10, No.5, pp. 515-525

Drugan, W. \& Willis, J. A. (1996). A micromechanics-based nonlocal constitutive equation and estimates of representative volume element size for elastic composites, Journal of the mechanics and physics of solids, Vol.44, No.4, pp.497-524

Dullien, F. A. L. (1979). Porous Media: Fluid Transport and Pore Structure, Academic Press Inc. San Diego, pp. 269-324

Fourie, W.; Said, R.; Young, P. \& Barnes, D. L. (2007). The simulation of pore scale fluid flow with real world geometries obtained from $x$-ray computed tomography, Proceedings of European COMSOL Multiphysics Conference, Boston, COMSOL inc.

Goel, A.; Tzanakakis, M.; Huang, S.; Ramaswamy, S.; Choi, D. \& Ramarao., B. (2002). Characterization of three-dimensional structure of paper using X-ray microtomography, Tappi Journal. Vol.84, No.5, pp. 111-122

Goel, A.; Arns, C. H.; Holmstad, R.; Gregersen, O. W.; Bauget, F., Averdunk, H.; Sok, R. M.;

Sheppard, A. P. \& Knackstedt, M. A. (2006). Analysis of the Impact of Papermaking Variables on the Structure and Transport Properties of Paper Samples by X-ray Microtomography, Journal of Pulp and Paper Science, Vol.32, No.2, pp. 111-122

Gonzales, R. C. \& Woods R. E. (2002). Digital Image Processing, second edition, Prentice Hall

Holmstad, R.; Aaltosalmi, U.; Ramaswamy, S.; Kataja, M.; Koponen, A.; Goel, A. \& Gregersen, O. W. (2005). Comparison of 3D structural characteristics of high and low resolution x-ray microtomographic images of paper, Nordic Pulp and Paper Research Journal, Vol.20, No.3, pp. 283-288

Holmstad, R.; Antonie, C.; Nygård P. \& Helle, T. (2003). Quantification of the threedimensional paper structure: Methods and potential. Pulp \& Paper Canada, Vol.42, No.7, pp. 86-189 
Kak, A. C. \& Slaney, M. (1988). Principles of Computerized Tomographic Imaging, IEEE Press Kass, M; Witkin, A. \& Terzopoulos, D. (1987). Snakes - Active Contour Models. International Journal of Computer Vision, Vol.1, No.4, pp.321-331

Koivu V.; Mattila K. \& Kataja M. (2009a). A method for measuring Darcian flow permeability. Nordic Pulp and Paper Research Journal, Vol.24, No.4, pp. 395-402

Koivu V.; Decain M.; Geindreau C.; Mattila K., Alaraudanjoki, J.; Bloch J.-F. \& Kataja M. (2009b). Flow permeability of fibrous porous materials. Micro-tomography and numerical simulations. Proceedings in the 14th Fundamental Research Symposium, Oxford, pp. 437-454.

Koivu V.; Decain M.; Geindreau C.; Mattila K.; Bloch J.-F. \& Kataja M. (2010) Transport properties of heterogeneous materials. Combining computerised X-ray microtomography and direct numerical simulations, International Journal of Computational Fluid Dynamics, Vol. 23, No.10, pp. 713-721

Kutay, M. E.; Aydilek, A. H. \& Masad, E. (2006). Laboratory validation of lattice Boltzmann method for modeling pore-scale flow in granular materials. Computers and Geotechnics, Vol.33, No.8, pp. 381-395

Leskelä, M. and Simula, S. (1998). Papermaking Science and Technology, Paper Physics, Transport phenomena, ISBN952-5216-16-0, Fapet Oy, Helsinki, pp. 284-317

Liakopoulos, A. C. (1965). Darcy's coefficient of permeability as symmetric tensor of second rank. Hydrolocical Sciences Journal, Vol.10, No.3, pp.41-48

Lundström, T. S.; Frishfelds, V. \& Jakovics, A. (2004). A statistical approach to permeability of clustered fibre reinforcements, Journal of Composite and Materials, Vol.38, No.13, pp. 1137-1149

Lorensen, W. E. \& Clive H. E. (1987a). Marching cubes: A high resolution 3D surface construction algorithm, ACM SIGGRAPH Computer Graphics, Vol.21, No.4, pp. 163169

Lorensen, W. E. \& Clive H. E. (1987b). Marching cubes: A high resolution 3D surface construction algorithm, Proceedings of the $14^{\text {th }}$ annual conference on computer graphics and interactive techniques, New York, USA

Manwart, C.; Aaltosalmi, U.; Koponen, A.; Hilfer, R. \& Timonen, J. (2002). Lattice-Boltzmann and finite-difference simulations for the permeability for three-dimensional porous media, Physical Review. E., Vol.66, No.1, 016702.

Martys, N. S. \& Hagedorn, J. G. (2002). Multiscale modeling of fluid transport in heterogeneous materials using discrete Boltzmann methods, Journal of Materials and Structures, Vol.35, No.10, pp. 650-659

Ojala, T.; Pietikäinen, M. \& Harwood, D. (1994). Performance evaluation of texture measures with classification based on Kullback discrimination of distributions, Proceedings of the 12th IAPR International Conference on Pattern Recognition (ICPR 1994), vol.1, pp. 582-585

Osher, S.; Sethian, J. A. (1988). Fronts propagating with curvature-dependent speed: Algorithms based on Hamilton-Jacobi formulations, Journal of Computational Physics, Vol.79, pp. 12-49

Perona, P. \& Malik J. (1987). Scale-space and edge detection using anisotropic diffusion. Proceedings of IEEE Computer Society Workshop on Computer Vision, pp. 16-22. 
Rasi, M., Koponen, A., Aaltosalmi, U., Timonen, J., Kataja, M., \& Niskanen, K. J. (1999). Permeability of paper: Experiments and numerical simulations. TAPPI, International Paper Physics Conference, pp. 297-306

Rolland du Roscoat, S.; Bloch, J.-F. \& Thibault, X. (2005). Synchrotron Radiation microtomography applied to paper investigation, Journal of Physics D: Applied Physics, Vol.38, pp. A78-A84

Rolland du Roscoat, S.; Decain, M.; Thibault, X.; Geindreau, C. \& Bloch, J.-F. (2007). Estimation of microstructural properties from synchrotron $X$-ray micro tomography and determination of the REV in paper materials. Acta Materiala, Vol.55, No.8, pp. 2841-2850.

Samuelsen, E. J.; Houen, P.-J.; Gregersen, O. W.; Helle, T. \& Raven C. (2001). Three Dimensional Imaging of Paper by Use of Synchrotron X-ray Microtomography, Journal of Pulp and Paper Science, Vol.27, No.2, pp. 50-53

Smith, S.M.; Brady, J.M. (1997). SUSAN - a new approach to low level image processing. Int. Journal of Computer Vision, Vol. 23, No.1, pp. 45-78

Stock, S. R. (2009). Micro Computed Tomography, Methodology and Applications, CRC press Taylor and Francis Group, Boca Raton

Thibault, X. \& Bloch, J.-F. (2002). Structural Analysis of X-ray Microtomography of a Strained Nonwoven Papermaker Felt, Textile Research Journal, Vol.72, No.6, pp. 480485

Tomasi, C. \& Manduchi, R. (1998). Bilateral Filtering for Gray and Color Images, Proceedings of the 1998 IEEE International Conference on Computer Vision, Bombay, IndiaTorquato, S. (2001). Heterogeneous Materials, Microstructure and Macroscopic Properties, Springer-Verlag New York Inc., New York, pp. 1-19

Verleye, B.; Croce, R.; Griebel, M.; Klitz, M.; Lomov, S.; Morren, G.; Sol, H.; Verpoest, I. \& Roose, D. (2007). Permeability of textile reinforcements: Simulation, influence of shear and validation, Composite Science and Technology, Vol.68, No.1, pp. 2804-2810.

Verleye, B.; Klitz, M.; Croce, R.; Roose, D.; Lomov, S. \& Verpoest, I. (2005). Computation of permeability of textile reinforcements. Proceedings in Scientific Computation IMACS 2005, Paris, France

Wiegman, A. (2007). Computation of the permeability of porous materials from their microstructure by fft-Stokes, Report of the Fraunhofer ITWM 129, Fraunhofer Institute

Wu, K.; Jiang, Z.; Couples, G. D.; Van Dijke, M. I. J. \& Sorbie, K. S. (2007). Reconstruction of multi-scale heterpogeneous porous media and their flow prediction, Proceedings in the International Symposium of the Society of Core Analysts, Calgary, Canada 


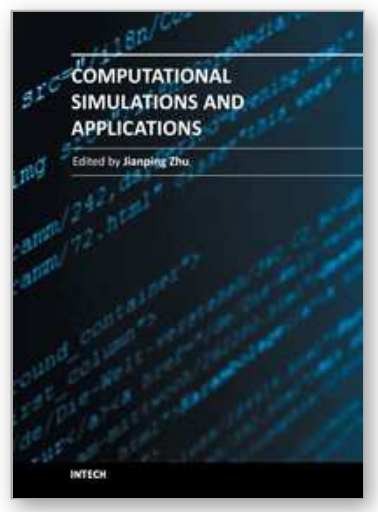

\section{Computational Simulations and Applications}

Edited by Dr. Jianping Zhu

ISBN 978-953-307-430-6

Hard cover, 560 pages

Publisher InTech

Published online 26, October, 2011

Published in print edition October, 2011

The purpose of this book is to introduce researchers and graduate students to a broad range of applications of computational simulations, with a particular emphasis on those involving computational fluid dynamics (CFD) simulations. The book is divided into three parts: Part I covers some basic research topics and development in numerical algorithms for CFD simulations, including Reynolds stress transport modeling, central difference schemes for convection-diffusion equations, and flow simulations involving simple geometries such as a flat plate or a vertical channel. Part II covers a variety of important applications in which CFD simulations play a crucial role, including combustion process and automobile engine design, fluid heat exchange, airborne contaminant dispersion over buildings and atmospheric flow around a re-entry capsule, gas-solid two phase flow in long pipes, free surface flow around a ship hull, and hydrodynamic analysis of electrochemical cells. Part III covers applications of non-CFD based computational simulations, including atmospheric optical communications, climate system simulations, porous media flow, combustion, solidification, and sound field simulations for optimal acoustic effects.

\section{How to reference}

In order to correctly reference this scholarly work, feel free to copy and paste the following:

Viivi Koivu and Tuomas Turpeinen (2011). The Effect of Tomography Imaging Artefacts on Structural Analysis and Numerical Permeability Simulations, Computational Simulations and Applications, Dr. Jianping Zhu (Ed.), ISBN: 978-953-307-430-6, InTech, Available from: http://www.intechopen.com/books/computationalsimulations-and-applications/the-effect-of-tomography-imaging-artefacts-on-structural-analysis-and-numericalpermeability-simulat

\section{INTECH}

open science | open minds

\section{InTech Europe}

University Campus STeP Ri

Slavka Krautzeka 83/A

51000 Rijeka, Croatia

Phone: +385 (51) 770447

Fax: +385 (51) 686166

www.intechopen.com

\section{InTech China}

Unit 405, Office Block, Hotel Equatorial Shanghai

No.65, Yan An Road (West), Shanghai, 200040, China 中国上海市延安西路65号上海国际贵都大饭店办公楼 405 单元

Phone: +86-21-62489820

Fax: $+86-21-62489821$ 
(C) 2011 The Author(s). Licensee IntechOpen. This is an open access article distributed under the terms of the Creative Commons Attribution 3.0 License, which permits unrestricted use, distribution, and reproduction in any medium, provided the original work is properly cited. 